\title{
An interactive contouring module improves engagement and interest in radiation oncology among pre-clinical medical students: Results of a randomized trial
}

\author{
Pushpa Neppala $^{1}$, Michael Sherer ${ }^{1}$, Grant Larson ${ }^{1}$, Alex Bryant ${ }^{1}$, Neil Panjwani ${ }^{1}$, James D. \\ Murphy ${ }^{1}$, and Erin F Gillespie ${ }^{1,2}$ \\ ${ }^{1}$ Department of Radiation Medicine and Applied Sciences, University of California San Diego, La \\ Jolla, CA
}

${ }^{2}$ Department of Radiation Oncology, Memorial Sloan Kettering Cancer Center, New York, NY

\begin{abstract}
Purpose-Studies have shown significant gaps in knowledge of radiation therapy among medical students and primary care providers. The goal of this study was to evaluate the impact of an interactive contouring module on knowledge and interest in radiation oncology among pre-clinical medical students.
\end{abstract}

Methods and Materials-Second year medical students at $* * *$ were randomized to participate in an interactive contouring exercise or watch a traditional didactic lecture on radiation oncology. Participants completed knowledge tests and surveys at baseline, immediately following the exercise, and 3 months later. Statistical analysis included Wilcoxon signed-rank test for pre- and post-test comparisons and Wilcoxon rank sum test for comparison between groups.

Results-Forty-three medical students participated in the trial (21 in the didactic group and 22 in the contouring group). Students completing the contouring module demonstrated similar overall knowledge improvement compared to the traditional didactic group ( $+8.6 \%$ vs. $+6.6 \%$, NS) but endorsed greater engagement on a 5-point Likert-type scale (3.10 vs 3.76, $\mathrm{p}=0.02)$. At 3-month follow-up, there was a non-significant trend toward improved overall knowledge in the contouring group ( $43 \%$ vs. $51 \%, p=0.10$ ), with a significance difference in a subset of questions on knowledge of the process of radiation therapy as well as side effects $(51 \%$ vs. $75 \%, \mathrm{p}=0.002)$. Students in the contouring group demonstrated more interest in pursuing a clinical radiation oncology rotation $(2.52$ vs $3.27, \mathrm{p}=0.01)$.

Conclusions-Use of an interactive contouring module was an effective method to teach preclinical medical students about radiation oncology, with no significant difference in knowledge

Correspondence to: Erin F. Gillespie, MD, Assistant Attending, Memorial Sloan Kettering Cancer Center, 1275 York Ave, Box 22, New York, NY 10065, Tel: 212-639-5868 Fax: 212-794-3188/ efgillespie@ucsd.edu.

Conflicts of Interest Notification

E.F.G., N.P. and J.D.M. are co-founders of the non-profit website eContour.org. There are no other conflicts of interest.

Publisher's Disclaimer: This is a PDF file of an unedited manuscript that has been accepted for publication. As a service to our customers we are providing this early version of the manuscript. The manuscript will undergo copyediting, typesetting, and review of the resulting proof before it is published in its final citable form. Please note that during the production process errors may be discovered which could affect the content, and all legal disclaimers that apply to the journal pertain. 
gained compared to a traditional didactic lecture. However, higher engagement among students completing the contouring module led to improved retention of knowledge of radiation side effects and greater interest in radiation oncology. These data suggest a potential benefit of integrating an interactive radiation oncology module into the pre-clinical medical school curriculum.

\section{Introduction}

More than half of all cancer patients will receive radiation therapy as part of their treatment(1), and there is an increasing number of patients that have received radiation among the 15.5 million cancer survivors in the United States today(2). Many types of physicians will help care for these patients as they live through cancer treatment and beyond, but recent studies indicate that physicians not specializing in radiation oncology have limited knowledge of radiation treatment and its sequelae $(3,4)$. A survey of Canadian family practitioners showed that the majority of respondents endorsed feeling very little to somewhat knowledgeable about radiation side effects, which correlated with performance on a knowledge-based test(5). Furthermore, physicians with lower overall knowledge scores were less likely to refer patients for radiation oncology when indicated, thereby reducing patient access to multidisciplinary care which improves outcomes in many cancer types. A separate study showed that completion of a clinical rotation in radiation oncology improved knowledge of radiation among primary care providers(3). However, limited access to radiation oncology clinics has led many schools to offer a 1-2 hour lecture on radiation oncology, while others do not include it in the curriculum at all(6-8). While a radiation oncology-specific lecture can improve student knowledge(9), a survey of UK students found that lectures were often too focused on technical details (such as radiation physics) that are not as clinically relevant to non-radiation oncology practitioners(10).

In recent years, experiential and interactive learning methods, including simulation(11) and games(12), have been explored to better appeal to millennial learners(13). Many of these efforts involve e-learning, which offers increased accessibility and personalization of educational content(14). E-learning modules have been shown to have similar efficacy to traditional lectures(15), including within the field of oncology(16).

We hypothesized that an interactive contouring module would be an effective method to teach medical students about radiation oncology. Here, we present the results of a randomized trial of second year medical students in which an interactive case-based contouring module was compared to a traditional didactic lecture.

\section{Methods and Materials}

\section{Recruitment}

All rising second year medical students (MS2) for the 2016-2017 academic year at *** received an email invitation to participate in a study to test a new educational tool for radiation oncology. Forty-three students enrolled in this study (out of 124 students enrolled in medical school at that time) and scheduled a 1.5-hour appointment in a research lab to complete the study. Medical students were compensated with a $\$ 30$ gift card upon study completion. The *** Institutional Review Board approved this study as exempt. 


\section{Trial design and intervention}

Students were randomized to watch a traditional didactic lecture (Didactic Group) or participate in an interactive contouring module (Contouring Group) as shown in Figure 1. Randomization was carried out in block format by date of initial study participation, and participants were not aware of their assignment until arriving to the research lab. Of note, all students then remained in the research lab for the duration of the exercise. Both the lecture and the module exposed participants to fundamentals of radiation therapy in addition to the role of radiation in the context of locally advanced cervical cancer. Both exercises were constructed and revised to address the same set of learning objectives for the exercise (Figure 2), which incorporated essential components or radiation oncology curriculum that had been previously identified(17). The Didactic Group watched a 40-minute lecture that was created to mimic the format and teaching style of a traditional lecture during the preclinical medical school curriculum. The Contouring Group worked through a PowerPointbased module(18) describing the case of a 45-year-old woman with FIGO stage IIB cervical cancer. The interactive contouring module followed the patient from diagnosis (including history and physical exam, biopsy, and imaging) through treatment (including multidisciplinary tumor board and chemoradiation) and survivorship. Within the module, students were instructed to contour the case (including the cervical mass and uterus, bladder, and rectum) on a set of anonymized DICOMs (including a CT scan with fused PET and empty structure set for contours) that had been uploaded to the MIM treatment planning system (MIM Software Inc., Cleveland, OH). To assist with contouring, students were provided access to the cervical cancer teaching case in eContour, which is a free web-based interactive contouring resource available online(19). The eContour case gives students the ability to scroll through 3-dimensional cross-sectional imaging and review relevant anatomy as it relates to radiation-related side effects.

\section{Knowledge and survey response analysis}

Both groups completed a baseline pre-test assessing knowledge of radiation oncology fundamentals, including several questions previously published by Zaorsky et al(3), as well as a survey including confidence with patient experience of radiation and female pelvic radiographic anatomy. Both groups also completed a post-test, which included these questions in addition to identification of radiographic anatomy of the female pelvis in a 3dimensional viewer. To assess validity of the testing instruments in the planning phase, the test was taken by 4 radiation oncologists outside the research team, and one question that was answered incorrectly was discarded. Additional survey questions were included in the post-test to assess engagement, usefulness, and likelihood of pursuing a clinical radiation oncology rotation using Likert-type scales, as previously described(20). Both groups also indicated at this time which type of cancer (breast, prostate, or lung) they would be most interested in learning about in a similar exercise. A follow-up retention test and survey was completed three months after the exercise, which again included both radiographic identification of female pelvic anatomy as well as fundamentals of radiation oncology including side effects. At this time, all students reviewed the cervical cancer case in eContour(19). Since all students had at this point completed their second-year oncology block (which occurred between the initial post-test and retention test), the survey inquired about the components that were lacking in their school's curriculum (based on learning 
objectives specified for the exercise under study, see Figure 2), and the extent to which this exercise addressed each learning objective. Additional survey questions included the importance of accessing radiographic imaging in 3D for learning anatomy and the likelihood of using eContour cases during the clinical oncology block. (See Supplementary Materials for all knowledge tests and surveys).

\section{Statistics}

Statistical analysis for all knowledge test results and most survey answers included Wilcoxon signed-rank test for pre- and post-test comparisons and Wilcoxon rank sum test for comparison between groups with a significance level of $a=0.05$. For two survey questions regarding the student's confidence that were repeated pre- and post-exercise (patient experience of radiation oncology and female pelvic anatomy), a difference-indifference technique was used. This involved determining the difference in confidence level on a Likert scale (where $1=$ Not at all confident and $5=$ Extremely confident) for each student between the pre- and post-test, and comparing this difference between the Contouring Group and the Didactic Group. Difficulty and discrimination index for pre-test, post-test, and 3-month follow-up calculated as previously described(21).

For the sample size analysis we assumed the null hypothesis was no difference in overall knowledge between the Contouring Group and Didactic Group (control), and the alternate hypothesis was that the students in the Contouring Group would improve overall knowledge scores by $20 \%$ (from $70 \%$ to $90 \%$, with predicted mean of $70 \%$ and SD of $20 \%$ at baseline) compared to the Didactic Group on the immediate post-test. Using a Wilcoxon rank sum test, a sample size of 32 would give an $80 \%$ power to detect a $20 \%$ improvement in overall knowledge for the Contouring Group ( $a=0.05$ ). We sought to enroll 45-50 second year medical students to account for subject dropout and uncertainties in mean and SD of baseline scores.

\section{Results}

All 43 medical students that enrolled participated in the trial. One student in the Didactic Group did not complete the pre-test, however all students completed the post-test and 3month follow-up. Both groups demonstrated similar baseline knowledge of radiation oncology on the pre-test (Table 1).

\section{Knowledge Assessment}

When compared to the baseline pre-test, the overall mean knowledge score of all participants on the immediate post-test was $+7.6 \%$ higher $(\mathrm{p}=0.007$ by Wilcoxon signed rank test). The improvement was similar in each of the two groups, with a mean score difference of $+8.6 \%$ in the didactic group and $+6.6 \%$ in the contouring module group. There were no significant differences between the groups (Table 1).

In a 3-month retention test, overall performance was not significantly different though a trend favoring the Contouring Group was noted ( $43 \%$ vs $51 \%$, $\mathrm{p}=0.10$ ). On subset analysis, the Contouring Group had significantly better knowledge of the radiation process and side effects $(51 \%$ vs $75 \%, \mathrm{p}=0.002)$ (Table 1$)$. 
Overall difficulty for the 3-month follow-up test was higher that the pre-test and initial posttest ( 0.86 vs 0.89 vs 0.42 , scale $0-1$ with 1 indicating easiest questions). This did result in an improved discrimination index for the follow-up test compared to the immediate post-test, indicating a better ability to identify high vs low performing respondents ( 0.14 vs 0.33 , $\mathrm{p}=0.02$; scale -1 to $+1,+1$ indicating most discriminatory questions). MCQs specifically addressing "RT process/side effects," were similar in both difficulty and discrimination compared to the "CT anatomy" subset. (See Supplementary Table 1 for results of each question and test).

\section{Engagement and Interest in Radiation Oncology}

On the immediate post-test, students in the Contouring Group endorsed greater engagement during the exercise on a 5-point Likert-type scale (3.10 vs 3.76, p=0.02) (Figure 3). Students in the Contouring Group were also more interested in completing a future clinical radiation oncology rotation (2.52 vs $3.27, \mathrm{p}=0.01)$. Both groups thought it would be useful to incorporate a similar exercise into the medical school curriculum, with a non-significant trend favoring the Contouring Group ( 3.45 vs $3.85, \mathrm{p}=0.11$ ). Gender concordance was noted in the type of cancer students would be most interested in learning about, should they complete another similar exercise. Specifically, $50 \%$ of males said they would select a prostate cancer case (vs 10\% of females) and 52\% of females chose breast cancer (vs $30 \%$ of males). There was a significant difference in disease type selected based on student gender $(\mathrm{p}=0.02)$.

\section{Method of learning 3-dimensional radiographic anatomy}

In the immediate post-test, students in the Contouring Group found the exercise more important for increasing their understanding of female pelvic anatomy compared to watching a lecture ( 2.71 vs $3.81, \mathrm{p}<0.001)$. Additionally, students in both groups demonstrated significantly increased confidence in CT-based pelvic anatomy compared to the pre-test survey (Figure 3), with a trend toward greater improvement in confidence among students in the Contouring Group (mean Likert score increase 0.9 vs 1.4, $\mathrm{p}=0.052$ ).

\section{Role of an interactive contouring module in the pre-clinical oncology block}

At 3-month follow-up, all students had completed the second-year oncology block. Among all students, CT-based anatomy was most often endorsed (74\% of students) as lacking in their current pre-clinical oncology curriculum, followed by multidisciplinary care (35\% of students) (Figure 4). When asked which learning objectives had been best addressed in the trial exercise, students from both the Didactic and Contouring Groups identified CT-based anatomy (67\% vs. $68 \%$, NS). There were trends suggesting that the Contouring Group learned more about multidisciplinary care $(77 \%$ vs. $52 \%$, $\mathrm{p}=0.09)$, while the didactic group learned more about radiation biology and physics ( $43 \%$ vs. $18 \%, \mathrm{p}=0.08$ ) (data not shown).

Also at 3-month follow up, all students (both groups) viewed the cervical cancer case in eContour. Students placed high importance on being able to scroll through patient imaging in 3D (Figure 5A) and more than half said they would be "quite likely" or "extremely likely" to use eContour cases during the second year oncology block (Figure 5B). 


\section{Discussion}

This randomized trial demonstrates the feasibility of using an interactive contouring module as a tool for introducing radiation oncology concepts to medical students. Students completing the module demonstrated similar improvements in overall knowledge to students watching a traditional didactic lecture, both immediately following the session and at 3month follow-up. This is consistent with other studies of e-learning in general oncology(22), including one randomized trial(23), which have shown equivalence compared to traditional techniques. However, on subset analysis of the 3-month follow-up test, the group that completed the contouring module demonstrated better retention of knowledge of radiation process and side effects.

While our initial hypothesis was that engaging students with case-based imaging and active contouring would enhance knowledge of CT-based anatomy, perhaps knowledge of the process and side effects of radiation are actually most critical for students not going into the field of radiation oncology, since they relate to counseling future patients about expectations of cancer treatment, possibly as early as in discussions about cancer screening. A recent survey of internal medicine residents found that only $50 \%$ of them would recommend stereotactic body radiotherapy (SBRT) for patients with medically inoperable early-stage lung cancer(24).

Previous work shows that students prefer web-based modules for image-intensive material(16). It is possible that the manipulation of images in the contouring module reinforced the location-based nature of radiation side effects. Students in the contouring group were more likely to state that the exercise was beneficial for learning CT-based anatomy and demonstrated a borderline significant increase in confidence in reading female pelvic CT scans. The majority of students also reported that CT anatomy was not well covered in their second year oncology block, and that they would be quite likely or extremely likely to use the eContour cases during their clinical clerkship, though there was potential for social desirability bias among study participants in answering these questions.

Students endorsed feeling more engaged through interactive learning, which may also explain greater long-term retention of side effects among the contouring group. Meanwhile, greater engagement may have led to the students' increased interest in pursuing a clinical radiation oncology rotation. Interactive learning could serve as an innovative method to expose medical students to radiation oncology earlier in medical school and increase interest in the field, since currently many students' first exposure occurs after residency choices have been made(6). Our finding that gender impacts a student's interest in cancer type is consistent with prior research about gender-concordant patient care $(25,26)$, but in this setting suggests customization of learning (as is available through e-learning) as a potential method to maintain student engagement while teaching general concepts such as multidisciplinary care and survivorship, which transcend cancer types.

Students in the contouring module group more often reported that the exercise taught them about multidisciplinary care, which students frequently endorsed as not being covered well in their second year oncology block. This is a common concern among undergraduate 
medical educators(27). In one study led by surgeons, the authors created and tested an interactive game to enhance students' understanding of multidisciplinary care in oncology(12). Our data suggest that an interactive contouring module can provide students with important information regarding the role of radiation as a component of a patient's cancer treatment.

Our study is unique for the randomized design, which allows for a direct comparison of interactive contouring to current teaching methods. Past studies of radiation oncology education programs have primarily investigated adding components to an existing curriculum $(6,28)$, which requires additional time and thus may be difficult to implement. Our study shows that an interactive module could represent a method to improve the educational experience and increase interest in radiation oncology without reducing knowledge or increasing the total dedicated teaching time. The module could be provided as an alternative for the subset of students to whom the interactive learning style appeals most. Meanwhile though, with many students not getting any exposure to radiation oncology through their pre-clinical curricula(7) this module offers an easy way to integrate additional information into the oncology block. An engaging and interactive introduction to radiation oncology earlier in medical school could encourage more students to participate in a clinical rotation, which also has proven benefits for long-term knowledge retention(3).

Limitations of the present study include first that the groups showed no significant difference in the primary endpoint, only in a subset analysis that was conducted post-hoc. The study was powered to detect a $20 \%$ improvement in knowledge compared to the control group, which was overly ambitious, given that difference in a prior study of a radiation oncology lecture improved knowledge by only 11\%(9). Furthermore, it was not anticipated that medical student performance on both the pre-test and immediate post-test would exceed $80 \%$. To increase the distribution of test scores on the follow-up test, a more difficult test was created, and a significant decrease in knowledge retention test scores was observed. This was despite completion of the second year pre-clinical oncology block in the interim. Perhaps future educational trials should consider long-term retention as the primary endpoint, since this likely correlates better with impact on clinical practice(29). It should also be noted that the study employed previously unvalidated knowledge tests. Negative phrasing was employed in a few questions, and, while this approach is generally not recommended, it can be employed to highlight a significant outcome or clinical practice. One such question involved identifying the responsibilities of a radiation oncologist, with the correct answer including all of the options except "pressing the button", which was selected specifically due to a prior publication showing that $23 \%$ of fourth year students answered this incorrectly(3). Though we attempted to assure construct validity, the possibility remains that our tests did not adequately assess radiation oncology knowledge. Lastly, this exercise required participants to be on site in order to use the MIM contouring software. However, our team is working towards an interactive online contouring application that would allow users to complete the module entirely online, providing increased access to education for medical schools without a radiation oncology department, or potentially in low-resource programs internationally. 
In conclusion, these data suggest that pre-clinical radiation oncology education, which impacts all physicians who will care for patients undergoing radiation and surviving after cancer treatment, may benefit from incorporation of interactive image-based contouring modules.

\section{Supplementary Material}

Refer to Web version on PubMed Central for supplementary material.

\section{Acknowledgments}

This work was supported by the National Institute on Aging, Grant T35 AG26757 (PI: Dilip V. Jeste, MD), and the Stein Institute for Research on Aging and the Center for Healthy Aging at the University of California, San Diego (to P.N.), as well as Radiological Society of North America Resident Research Seed Grant (to E.F.G.).

\section{References}

1. Delaney G, Jacob S, Featherstone C, et al. The role of radiotherapy in cancer treatment: Estimating optimal utilization from a review of evidence-based clinical guidelines. Cancer. 2005; 104:1129-37. [PubMed: 16080176]

2. Miller KD, Siegel RL, Lin CC, et al. Cancer treatment and survivorship statistics, 2016. CA Cancer J Clin. 2016; 66:271-89. [PubMed: 27253694]

3. Zaorsky NG, Shaikh T, Handorf E, et al. What are medical students in the united states learning about radiation oncology? Results of a multi-institutional survey. Int J Radiat Oncol Biol Phys. 2016; 94:235-42. [PubMed: 26684409]

4. Samant R, Malette M, Tucker T, et al. Radiotherapy education among family physicians and residents. J Cancer Educ. 2001; 16:134-8. [PubMed: 11603874]

5. Olson RA, Lengoc S, Tyldesley S, et al. Relationships between family physicians' referral for palliative radiotherapy, knowledge of indications for radiotherapy, and prior training: A survey of rural and urban family physicians. Radiat Oncol. 2012; 7:73. [PubMed: 22607650]

6. Dennis KE, Duncan G. Radiation oncology in undergraduate medical education: A literature review. Int J Radiat Oncol Biol Phys. 2010; 76:649-55. [PubMed: 20159359]

7. Jagadeesan VS, Raleigh DR, Koshy M, et al. A national radiation oncology medical student clerkship survey: Didactic curricular components increase confidence in clinical competency. Int J Radiat Oncol Biol Phys. 2014; 88:51-6. [PubMed: 24331651]

8. Clayton R, Trotter T. The impact of undergraduate education in radiation oncology. J Cancer Educ. 2013; 28:192-6. [PubMed: 23436001]

9. Hirsch AE, Mulleady Bishop P, Dad L, et al. An increase in medical student knowledge of radiation oncology: A pre-post examination analysis of the oncology education initiative. Int $\mathbf{J}$ Radiat Oncol Biol Phys. 2009; 73:1003-8. quiz 1008.e1-1008.e2. [PubMed: 19251088]

10. Cave J, Woolf K, Dacre J, et al. Medical student teaching in the uk: How well are newly qualified doctors prepared for their role caring for patients with cancer in hospital? Br J Cancer. 2007; 97:472-8. [PubMed: 17667931]

11. Khan K, Pattison T, Sherwood M. Simulation in medical education. Med Teach. 2011; 33:1-3. [PubMed: 21182376]

12. Fukuchi SG, Offutt LA, Sacks J, et al. Teaching a multidisciplinary approach to cancer treatment during surgical clerkship via an interactive board game. Am J Surg. 2000; 179:337-40. [PubMed: 10875998]

13. Kasprzak T. Technology and radiology education-meeting the needs of millennial learners. Acad Radiol. 2016; 23:844-7. [PubMed: 27118526]

14. Ruiz JG, Mintzer MJ, Leipzig RM. The impact of e-learning in medical education. Acad Med. 2006; 81:207-12. [PubMed: 16501260] 
15. Davis J, Crabb S, Rogers E, et al. Computer-based teaching is as good as face to face lecture-based teaching of evidence based medicine: A randomized controlled trial. Med Teach. 2008; 30:302-7. [PubMed: 18484458]

16. Mehta MP, Sinha P, Kanwar K, et al. Evaluation of internet-based oncologic teaching for medical students. J Cancer Educ. 1998; 13:197-202. [PubMed: 9883777]

17. Oncology Education Committee. Ideal oncology curriculum for medical schools. Sydney: The Cancer Council Australia; 2007. http://www.cancer.org.au//Healthprofessionals/ OncologyEducation/IdealOncology.htm [Accessed 9/3/17]

18. Gillespie E. eContour.org. La Jolla, CA: 2016. Cervical cancer module. https://econtour.org/ fundamentals/cervicalcancer [Accessed 9/3/17]

19. Gillespie E. eContour.org. La Jolla CA: 2016. Case 64: Cervical cancer. https://econtour.org/ cases/64 [Accessed 9/3/17]

20. Sullivan GM, Artino AR. Analyzing and interpreting data from likert-type scales. J Grad Med Educ. 2013; 5:541-2. [PubMed: 24454995]

21. Gajjar S, Sharma R, Kumar P, et al. Item and test analysis to identify quality multiple choice questions (mcqs) from an assessment of medical students of ahmedabad, gujarat. Indian J Community Med. 2014; 39:17-20. [PubMed: 24696535]

22. Gaffan J, Dacre J, Jones A. Educating undergraduate medical students about oncology: A literature review. J Clin Oncol. 2006; 24:1932-9. [PubMed: 16622269]

23. Miedzybrodzka Z, Hamilton NM, Gregory H, et al. Teaching undergraduates about familial breast cancer: Comparison of a computer assisted learning (cal) package with a traditional tutorial approach. Eur J Hum Genet. 2001; 9:953-6. [PubMed: 11840198]

24. Shaverdian N, Yoo SM, Cook R, et al. Gaps in radiation therapy awareness: Results from an educational multi-institutional survey of us internal medicine residents. Int J Radiat Oncol Biol Phys. 2017; 98:1153-1161. [PubMed: 28721899]

25. Lurie N, Slater J, McGovern P, et al. Preventive care for women. Does the sex of the physician matter? N Engl J Med. 1993; 329:478-82. [PubMed: 8332153]

26. Ramirez AG, Wildes KA, Nápoles-Springer A, et al. Physician gender differences in general and cancer-specific prevention attitudes and practices. J Cancer Educ. 2009; 24:85-93. [PubMed: 19431022]

27. Matkowski R, Szelachowska J, Szewczyk K, et al. Improvements in undergraduate oncology education introduced at polish medical universities between 2004 and 2010 under poland's "National program for combating neoplastic diseases". J Cancer Educ. 2014; 29:428-33. [PubMed: 24627083]

28. Golden DW, Braunstein S, Jimenez RB, et al. Multi-institutional implementation and evaluation of a curriculum for the medical student clerkship in radiation oncology. J Am Coll Radiol. 2016; 13:203-9. [PubMed: 26410347]

29. Butler AC, Raley ND. The future of medical education: Assessing the impact of interventions on long-term retention and clinical care. J Grad Med Educ. 2015; 7:483-5. [PubMed: 26457162] 


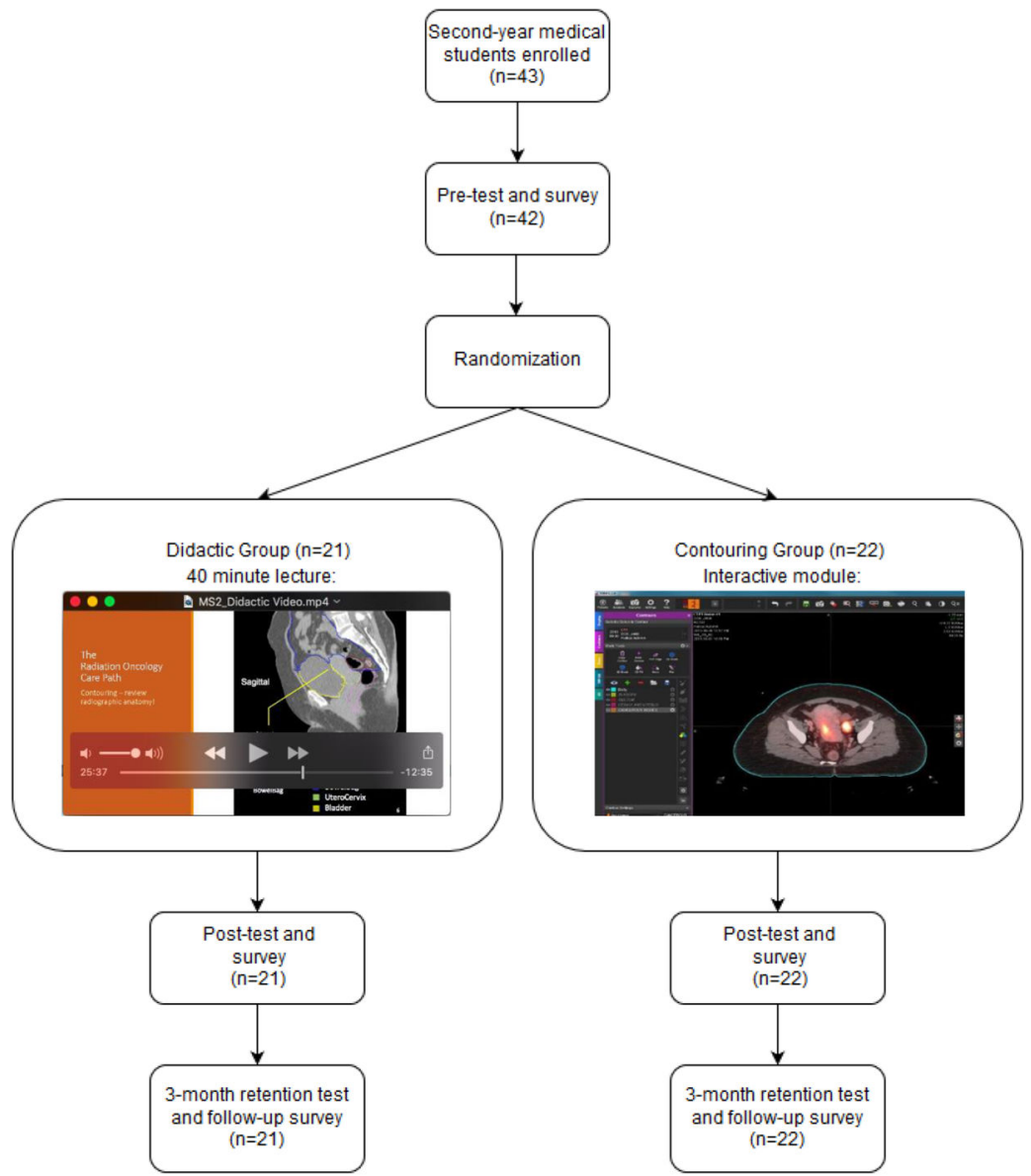

Figure 1. Randomized trial schema

The image in the "Didactic Group" shows a slide teaching pelvic imaging in the videorecorded didactic lecture. The image in the "Contouring Group" box shows the cervical cancer case students contoured in the MIM treatment planning software. 
Learning Objectives

1. General management of the cancer patient

a) Describe multidisciplinary care

b) Identify role of PET in cancer staging

c) Describe the role of the radiation oncologist in treatment

d) Describe the goal of radiation therapy

2. Understand the patient experience during radiation treatment

3. Understand the importance of anatomy in radiation treatment planning

a) Understand the purpose of target delineation (contouring)

b) Understand basic female pelvic anatomy and which organs are at risk of injury

4. Understand long-term side effects of radiation therapy for cervical cancer

5. Describe the incidence of cervical cancer and methods of cervical cancer prevention

Figure 2. Radiation oncology learning objectives

The objectives used for this pre-clinical radiation oncology educational exercise. 


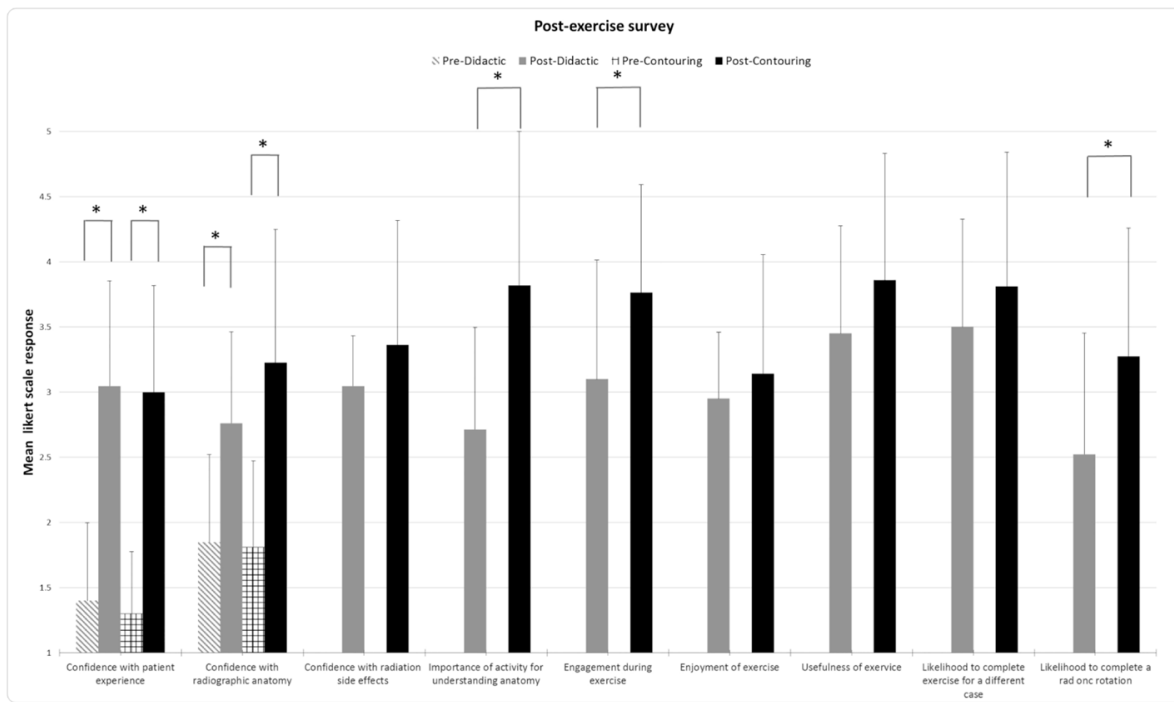

Figure 3. Responses to survey questions using 5-point Likert-type scale

All questions were asked on the post-exercise survey. Only the first 2 questions regarding confidence were asked on both the pre-test and post-test. For all post-exercise questions $\mathrm{N}=22$ for the contouring module group and $\mathrm{N}=21$ for the didactic group. For all pre-test questions, $n=22$ for contouring group responses and $n=20$ for didactic group responses. Markers $(*)$ indicate a significant difference $(\mathrm{p}<0.05)$. 


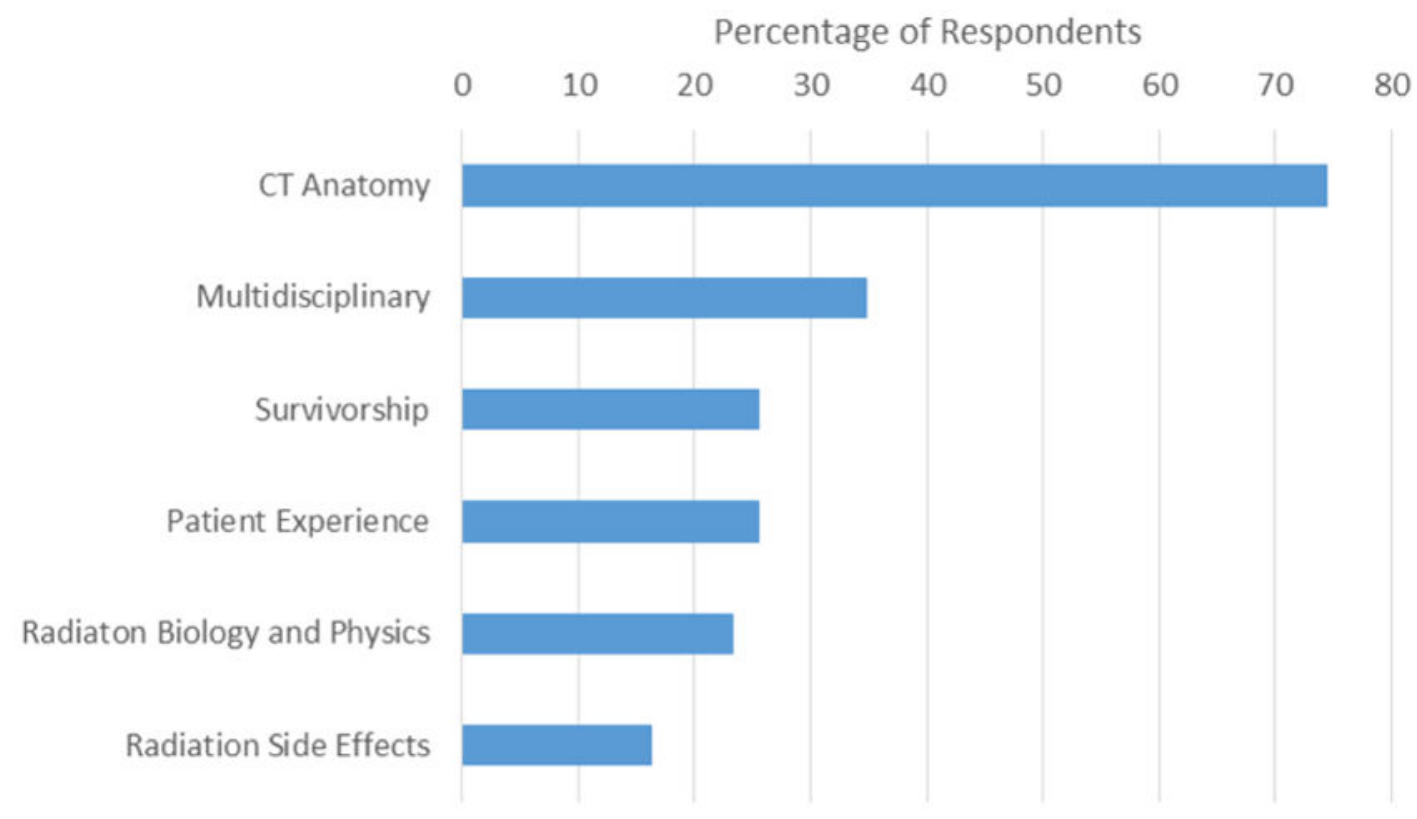

Figure 4. Gaps in the second year oncology curriculum

The percentage of respondents $(n=43)$ who selected each learning objective (as defined in the exercise under study) as being "least well covered in their oncology block". 


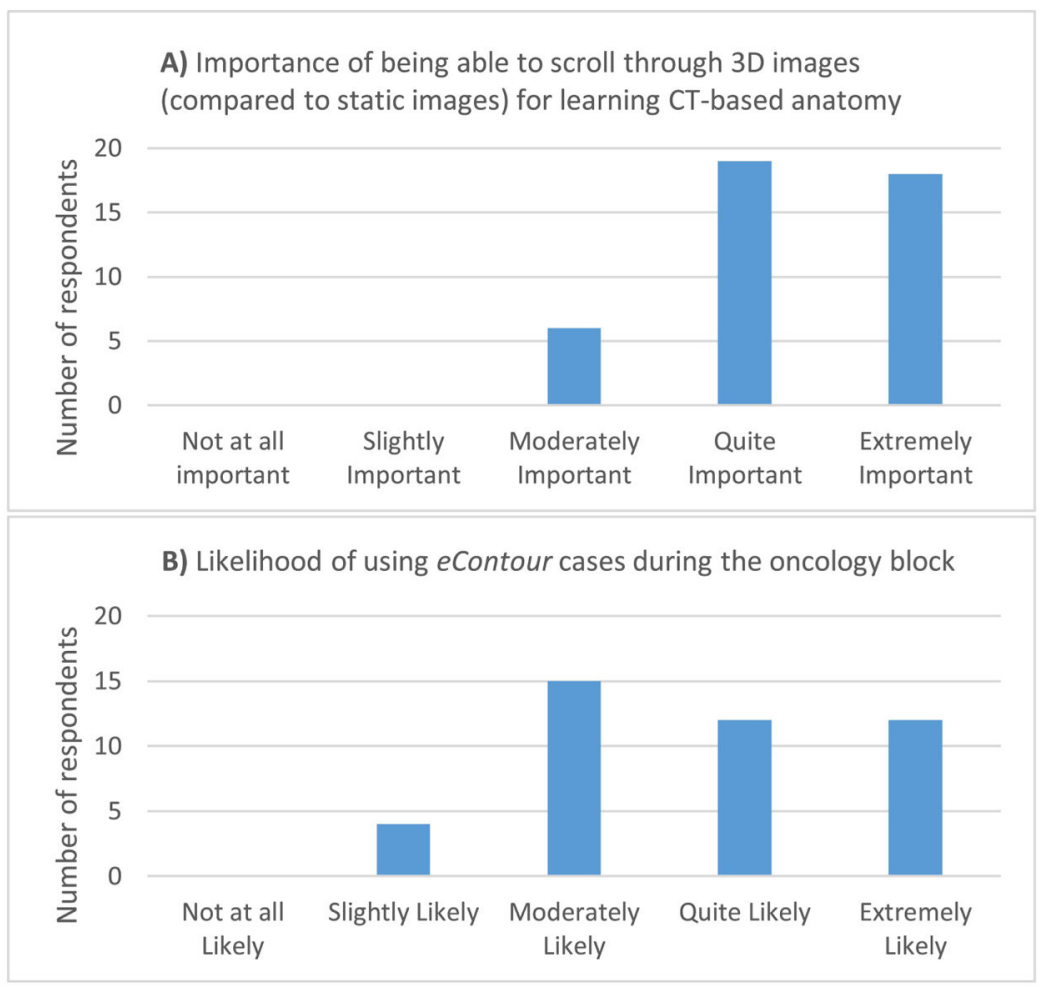

Figure 5. Utility of interactive 3D CT-based images

Parts A and B show the number of respondents $(n=43)$ for each option on the 5-point Likerttype scale for 2 separate questions asked of all students on the 3-month follow-up. 


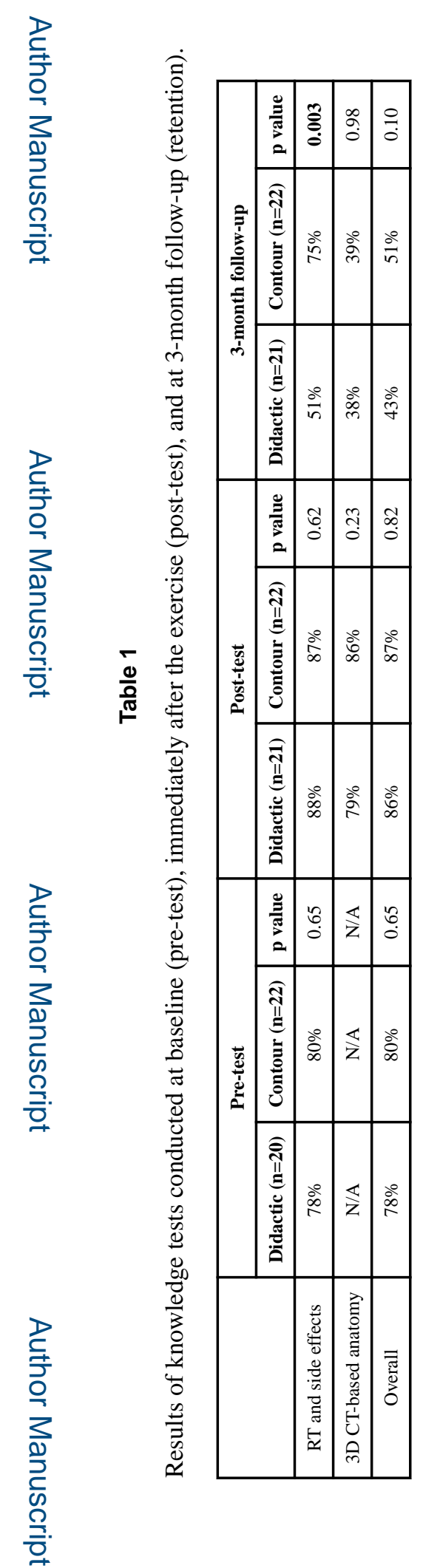

Pract Radiat Oncol. Author manuscript; available in PMC 2019 July 01. 